\title{
Lapurdum
}

LAPURDUM Euskal ikerketen aldizkaria | Revue d'études basques |

Revista de estudios vascos | Basque studies review

$5 \mid 2000$

Numéro $\mathrm{V}$

\section{Oi arnoaren goxoa! ou des « nominaux » du basque}

Jacques Allieres

\section{OpenEdition \\ Journals}

Édition électronique

URL : http://journals.openedition.org/lapurdum/1365

DOI : $10.4000 /$ lapurdum. 1365

ISSN : 1965-0655

Éditeur

IKER

Édition imprimée

Date de publication : 1 octobre 2000

Pagination : 383-390

ISBN : 2-84127-161-7

ISSN : $1273-3830$

\section{Référence électronique}

Jacques Allieres, « Oi arnoaren goxoa! ou des «nominaux » du basque », Lapurdum [En ligne], 5 | 2000, mis en ligne le 01 juin 2009, consulté le 21 septembre 2020. URL : http://journals.openedition.org/ lapurdum/1365; DOI : https://doi.org/10.4000/lapurdum.1365 


\title{
Oi arnoaren goxoa! ou des « nominaux » du basque
}

\author{
t Jacques Allières
}

Cet article de Jacques Allières n'avait pu être inséré dans le Lapurdum $I V$ en hommage à Jean Haritschelhar, et ce pour des raisons techniques ; nous le publions ici comme complément à ce Lapurdum $I V$. Par la même occasion il avait envoyé à la rédaction de la revue la lettre suivante, dans laquelle il s'inquiétait de l'une de ses étudiantes japonaises qui devait soutenir une thèse, et faisait part également d'ennuis de santé qui paraissaient alors bénins. Sa lettre témoigne du modèle de basque « navarro-labourdin litteraire » qu'il affectionnait :

Ipar-Tolosa'n, 1999 'ko urriarren 23'garrenean,

Adixkide maitea,

Horra, hitzemana bezala, nere lanttoa, Joanes Haritschelhar jaunaren omenaldiarentzat egina. Eztakit hortaz zer pentsatuko duzun, ez eta 're eian " aski urrun » doan... Bainan aspaldi danik « ateratzeko » beharra nuen...

Egia erran, emaiten dudan hitzen andana sobera luzea dela uste dut : beharbada horrialde bakotxean bi lerrotan zati laiteke, bata ezker, bertzea eskuin : ez lezake sobera paper " jan »... Dena dela, zuk ikus!

Damu handi bat egiten daut Euskal-Herrirako piai ttipiaren " erortzerat uzteak" ! Bainan sobera lan, eta nere ezkerreko zangoa oraino minbera...

Bihotzaren erditik agur bero bat - eta nere Japon'eko istudiantsaz (?) urrikal, otoi !

Zure,

J. Allières

Le petit vignoble d'Irouléguy, mûrissant sur ses collines ensoleillées qui séparent en Basse-Navarre le pays de Cize du pays de Baïgorry, nous réservait jadis une fort intéressante constatation : lors d'une visite à la coopérative, on pouvait lire en effet sur les étiquettes vantant -en basque- les qualités du nectar local $\mathrm{O} i$ arnoaren goxoa!, ce qui devait signifier en français « ô la suavité du vin'». Or si des énoncés tels que arno hori goxoa da « ce vin est agréable », ou badugu hemen arno goxo bat « nous avons ici un vin agréable» nous étaient familiers, en revanche l'étiquette en question récélait à nos yeux, par la structure de cette courte formule, une énigme à dénouer, puisque la traduction mot-à-mot devait être «Ô le suave du vin ! ». 
Dans nos langues romanes, et même indo-européennes sauf exception (anglais par exemple), le propre d'un adjectif, indépendamment de sa sémantique, consiste dans sa variabilité - plus ou moins générale - en genre : phonétiquement bon bonne, doux douce, oisif oisive, plaisant plaisante, vieux vieille etc., et graphiquement souvenir d'une ancienne prononciation effective-nu nue, pur pure, seul seule etc., les vrais épicènes, sur les deux plans du son et de la graphie, étant évidemment les adjectifs terminés par un -e au masculin : agréable, timide, utile, vide etc. En Europe, outre l'anglais, seules les langues ouraliennes (finnois, lapon, estonien, hongrois), le turc et... le basque ignorent ce trait caractéristique.

Une autre construction fondamentale de cette dernière langue fait néanmoins d'elle un cas spécifique de "confusion » entre substantif et adjectif. On sait en effet que dans cet idiome le déterminant précède généralement le déterminé : aitaren semea 'le fils du père', itsasoko urak 'les eaux de la mer' etc. Or, si le latin construisait parallèlement patriis filius, maris aquú et dulce vinum, il n'en va pas de même en basque, où l'on ne peut dire dans ce dernier cas que arno goxoa. Cette sorte de contradiction a fait élaborer bien des développements et des théories. Parmi celles-ci, la proposition d'André Martinet nous paraît digne d'attention, qui suggère que 1 adjectif est en fait un « nominal » qui ne se distingue du substantif que secondairement. Ainsi, arno goxoa ne signifierait pas à l'origine « le vin agréable » mais bien « l'agrément du vin ». Et tout rentre dans l'ordre.

On peut du reste alléguer pour appuyer cette interprétation le cas parallèle des tournures etxe aintzinean et etxearen aintzinean « devant la maison » : en effet, si la première juxtapose deux « nominaux » en construisant la détermination par simple antéposition du déterminé, tandis que la seconde la marque expressément par l'emploi du génitif antéposé, nous avons là comme un écho du couple arno goxoa et arnoaren goxoa, où la traduction française distingue « le vin agréable » - première construction - et «l'agrément du vin » seconde construction qui motive précisément mon article. La valeur des deux tournures n'est-elle pas la même?

La solution de ces difficultés ne résiderait-elle pas dans une perspective qui confondrait les prétendus « adjectifs » et "substantifs » sous la notion unique de " nominaux »? Malheureusement, on ne peut exprimer cette " neutralisation » d'une opposition dans une langue telie que le français où cette opposition est intransigeante ! Bien entendu! la sémantique commande les emplois « adjectivaux » et « substantivaux » de ces nominaux : etxe « maison » ne peut s'employer que « substantivement », gazte « jeune » qu' « adjectivement». Mais une foule d'autres cas manifestent cette propriété comme nous le verrons tout à l'heure.

Allons plus loin : nous ne prendrons qu'un seul exemple, un peu au hasard, celui de bazter. Le terme signifie selon le dictionnaire de Lhande d'une part « extrémité, étendue, bord, rivage » etc., et de l'autre « écarté », ex. ezta lekhü hañ bazterrik « il n'est si lieu si écarté », mais le verbe « dérivé » baztertu « écarter », etc. ne permet-il pas une construction bazter dezagun, «écartons-le », où ce même bazter, déjà adjectif et substantif, assume comme " infinitif radical » (terminologie de P. Lafitte), une fonction verbale ? La situation du basque se rapprocherait ainsi de celle du chinois - ou de l'anglais, curieusement-, où un même terme peut selon 
sa place relative dans l'énoncé « être » adjectif, substantif, verbe ou - bien sûr adverbe !... Adopter cette perspective conduirait peut-être à de nouvelles analyses de la composition et de la suffixation en basque.

Une enquête lexicale dans le dictionnaire précité va nous permettre d'apprécier la fréquence des cas où un même lexème peut jouer les deux rôles, celui d'adjectif et celui de substantif, plus celui d'adverbe, souvent, et même un quatrième, celui de prédicat verbal dans les limites susdites.

Voici la liste (il peut y avoir des oublis et des cas discutables) de ces items nous respectons l'orthographe de Lhande, marquant d'un astérisque comme lui les termes d'origine vraisemblablement romane, et imprimant en grasses ceux qui ne donnent pas lieu à une dérivation verbale (celle-ci peut se faire sur un dérivé simple, v. ci-après. Souvent le lexème non dérivé est d'origine romane) ; nous ne citons pas tous les équivalents français :

Aiher « animosité, soupçon »/« porté vers »+-tu,

*aisa « aisance »/《 aisé »/《 aisément 》 (soul. aisatü « devenir facile »),

antsi « souci $» / \ll$ indifférent» $+-t u$,

apho « crapaud »/« faible de caractère » $+-t u$,

aphur « de petite quantité »/« petite quantité »»+-tu,

argi « lumière »/« clair »+-tu,

ari « actif $» /(=$ aria $)$ « rapport $» / 《$ agir $»+-t u($ rare $)$,

*arrai « rayon »/《 rayonnant $»+t u$,

arrail " gros éclat de bûche $» /[$ employé adverbialement !] $+-d u$,

arrotz « étrange (r) »/《étranger »+-tu (arroztu),

ase « repu, ample »/《 repas surabondant $» / \ll$ se rassasier $»+(-t u)$,

asti « loisir »/« lent»,

asturu (arch.) « sort »/« malheureux »,

azken « dernier $» /$ « fin, terme » $+-d u,-t u$,

barhen « extrémité »/« profond»,

basa II-III « sauvage »/« lieu désert », +tu,

*baxo « bas »/《 fond »,

bazter « rivage $» /$ écarté »+-tu,

behar « besoin »/«pauvre» $+-t u$,

bello « tiède $» / 4$ chaleur",

bero $«$ chaleur $» / \ll$ chaud $»+-t u$,

berri « nouvelle $» / \ll$ neuf $»+-t u$,

bethe «(le) plein » (ex. ahurraren bethea)/ « plein » = Ppass,

bizi « vie »/« vif, vivant », = Ppass,

bizkar « dos »/« onéreux » (?),

bühür « tordu »/《 caractère tordu de qqch $»,+-t u$

bulunba « clarine »/« nigaud », + -tu,

busti, « mouillé, humide $» / \ll$ humidité $=$ Ppass ou $+-t u$,

damu « dommage $» / \ll$ sensible $»+-t u$,

*desarau « dérèglement »/ « extravagant »,

dohakabe « malheureux »/ « malheur» + -tu, 
doi « contenance $» / \ll$ exact $)+-t u$,

dollor « méchant $» / \ll$ peine, chagrin $»+-t u$,

egarri « soif $» / \ll$ altéré $»+-t u$,

egoki « habitant »/《 propre, convenable»

ekai « matière »/propre à être fait »,

elge « champ, plaine »/« plat, uni »,

eme « femelle »/féminin »+ -tu,

enpatxu « grossesse d'une fille ou d'une veuve $» / \ll$ enceinte $»$ (adj.) + - (a)tu, entregu, « adroit $» /$ remise, commission $»+-(a) t u$,

erakaitz, « mauvaise grâce ; difformité $» / \ll$ maussade ; difforme » +-tu, ztu), erdi « moitié »/《 demi » + (-tu) « accoucher, mettre bas »,

eri « maladie $» / \ll$ malade $»+-t u$,

erkhide « comparable, commun »/« coparticipation, cheptel »+ -tu,

errai, « entraille »/《 enragé »

erre $\langle$ colère $» /\langle$ ardent $»=$ Ppass,

erromes « déguenillé »/« pèlerin » $+-t u$, « appauvir ».

erskan « fort $» / \ll$ force (cf. erskondu « fortifier »),

eskas « insuffisant $» /\langle$ insuffisamment $($ adv. $) /\langle$ insuffisance $»+-t u$,

ezdeus «(le) néant $» / 《$ inutile »+ -tu,

ezti « miel $» /\langle$ doux $» /\langle$ doucement $»+-t u$,

gai « matière, sujet »/《 capable $»+-t u$,

gaihen « supérieur $» / \ll$ nécessité $»+-d u$,

gaitz « mal, maladie $» / \ll$ mauvais, difficile $»+-t u$ (gaiztu),

gaizki «(le) mal »/« mal en point »/« mal » (adv.) + -tu,

* gano « aptitude »/《 opportun »,

garai « sommet »/《 élevé» + -tu,

garbal « chauve $» / \ll$ calvitie » $+-d u$,

garhi « mince »/《 maigreur »+ -tu,

gar « flamme »/« brûlant » $+-t u$,

gauza « chose $» /$ excellent $»(?)$,

gaxo « malade $» / \ll$ maladie $»+-t u$,

geldo « débris $» / «$ faible $»+-t u$,

gelpe « lourdaud $» / \ll$ stupidité », *gereño « indécis »/《étalon (cheval) »,

* gerthu « hasard »/《 assuré » (?),

gibel $« \operatorname{dos} » / \ll$ loin $» / \ll$ timide $»+-d u$,

ginarri « substance $» /\langle$ membru »,

gizen " gras (se) $» / \ll$ (le) gras » $+-d u$,

goait 《 attente $» / \ll$ preste $» / \ll$ aux aguets $»+-(a) t u$,

gogurri « manque d'énergie »/« lâche »+ -tu,

goi $«$ hauteur $» / \ll$ haut $»+-t u$,

goibel « obscurité »/《 obscur»+-du,

goiphe « gras(se) »/ " graisse » (cf. koipatu),

gorde « caché, secret $» / \ll$ abri » $+-t u$,

gose « affamé $» / \ll$ faim » $+-t u$,

goxo « doux »/《douceur»+ - (a)tu, 
gozo « saveur »/《 doux » + -(a)tu,

gura « désir, volonté »/désireux de »+ -tu,

gur $(h) i$ 《 graisse $» / \ll$ gras $(\mathrm{se}) »+-i u$,

(h) aize « vent $» / \ll$ arrogant, sauvage $»+-(a) t u$,

*hastio " dégoût »/《 dégoûtant »+ -tu,

hauta « choix »/《 excellent »+ -tu,

herabe « répugnance, timidité $» / \ll$ timide $»+-t u$,

*herrebes « revers de la main »/〈( maladroit »,

hersi « clôture »/《 étroit», = Ppass (hertsi),

heze « humide $» / \ll$ humidité $+-t u$,

hil $《 \operatorname{mort}(\mathrm{e}) » / \ll \operatorname{mort} »=$ Ppass,

*hira « colère $» / \ll$ haineux »+ -tu,

hondar « fond $» / \ll$ dernier, qui reste $»,+-t u$,

hordi $\langle$ barrique $» / \ll$ ivrogne $»+-t u$,

idi « bouf »/« châtré »,

idor $« \sec » /\langle$ terre, continent $»+-t u$,

iduri « ressemblance $» / \ll$ semblable $»+-t u$,

igeri « nage $» / \ll$ nageant, en nage $»+-t u$,

ikhara « tremblement $» / \ll$ tremblant »+ $-t u$,

ilain « bout de laine etc. »/« frêle, vain $»+-d u$,

ilhaun « fétu, balle »/faible + -d $t u$,

ilhun « obscurité »/《 obscur» $+-d u$,

indargabe « faible »/faiblesse » $+-d u$,

irakin « bouillant $» / \ll$ bouillonnement $»=$ Ppass irakitu

ixtil « boue »/《 malpropre »+ $+d u$,

izerdi « sueur $» / \ll$ trempé de sueur »+ -tu,

izor « enceinte, grosse »/《 faculté de procréer »+-(a)tu (izorratu),

izpi « brin »/《 menu » (v. izpikatu),

izurri « infection, puanteur »/« sale »+-tu,

jauzi « bond $» /$ bondissant $»=$ Ppass,

jende « famille $» /\langle$ poli, urbain » $+-t u$,

jori $\ll$ abondant $» / \ll$ abondance $»+-t u$,

kadura « long; hésitant »/《 doute »+-tu,

kalaka « traquet, crécelle »/« bavard »+ -tu,

kalpar « chauve »/ « touffe de cheveux »,

kalte « dommage, perte »/《 nuisible»

*kara « mine, air »/« conforme»,

kharats « fétide »/《 puanteur»,

kharmin « âcre, piquant »/《 amertume, fiel »+-du,

* khexa « inquiétude, empressement »/« rapide, empressé »+-tu,

*khexu « inquiet »/inquiétude »

khitzika 《excitation »/《 excitant »+ -tu,

khopor « coupe $» / \ll$ concave, rond », khordoka « vacillation »/《ébranlé + -tu, khorotz « fiente $w / \ll$ fainéant » 
khozu 4 contagion $» /$ « contagieux »,

kide « égal »/« compagnon» $+-t u$,

kilika « chatouillement »/《 chatouilleux »+ -tu,

kiskail « croûte d'un objet rôti »/« trop rôti » +-du,

konkor « bosse »/« bossu »+-tu,

kopa « gobelet »/« creux, arrondi »+-tu,

korka « bosse »/《 bossu »+-tu,

koxkor « croûtọn »/《 trapu »,

küxka « assoupissement »/《 dodelinant de la tête de sommeil »,

laket « plaisant »/

lanbo « brouillard »/« obscur » $+-t u$,

$*$ langi « langueur $» / \ll$ languissant $»+-t u$,

*largo « largeur »/large »,

larre « lande »/« grossier, rustre»,

larri « volumineux »/« grosse pièce (d'argent)» $+-t u$,

laster « course »/ « rapide $/ /$ « vite » (cf. lasterkatu),

latz « rude, âpre, sévère »/《e effroi »+-tu (sic),

lauso « atome, corpuscule, bourrasque de neige »/« myope »+ -tu,

*laxo « lâche, détendu, desserré »/《état de ce qui est détendu »+-tu, (cf. laxatu),

laztan « soigneux »/《 soin »,

legar « impôt »/《légal »+-tu, (« devenir/rendre caillouteux », cf. legar « galet »), leka « bave »/《 traînant, humide »+-tu,

lerra « glissant $» /\langle$ glissade $»+-t u$,

likhits " dégoûtant »/《 saleté »+-tu, (kikistu/-tatu),

$*$ limuri « suintement, plaisir »/« glissant, flatteur »+ $+-t u$,

liskar « dispute $» / \ll$ tenace $»+-t u$,

lizun « moisissure $» / \ll$ moisi » $+-t u$,

lo « sommeil »/« endormi »+ -tü,

logale « envie de dormir »/《 qui a envie de dormir »+ -tü,

lohi « boue »/《 malpropre» $+-t u$,

lore « fleur »/« tranquille »+ -tu,

lots $a$ « crainte $» /$ craintif $»+-t u$,

lotsagabe « hardi »/《 intrépidement »+ -tu,

mahel « étang »/« calme»

*maingu « boiteux »/ « boiterie » + -tu, maite « aimé »/《 amour» (cf. maitatu), makhur « tordu »/《 adversité $»+-t u$, malkar « escarpé »/rocher, précipice marfundi « rhume »/« enrhumé + -tu, marranta « rhume »/ « enrhumé »+-tu, mehar « étroit »/« rétrécissement »+ -tu, $m e n$ 《 autorité $》 /\langle$ soumis » (cf. menpetu), *mengo « privation »/《 nécessiteux » (cf. mengoatu)

*men $(t) s$ « manque »/《 idiot»+-tu (menstu), min « mal, souffrance $» / \ll$ dur, piquant, intime (ami) »+-tu/-du, 
mokor « motte, éminence »/《 engourdi, rude » $+-t u$, motz « court »/《 parties sexuelles féminines » +-tu (moztu), mozkor « ivre »/« ivresse »+ -tu, muker « dur »/《 tristesse »+ -tu, mukurru « plénitude »/《 qui déborde » $+-t u$, naba « grande plaine »/ « plan, uni », nagi « paresseux $» / \ll$ paresse $»+-t u$, na (g) usi « maître »/《 important, « précieux »+-tu, naharo « abondance $» / \ll$ abondant $» /$ abondamment $»+-t u$, nahigabe « (de caractère) mou »/《 déplaisir »+ $t u$, nakhaitz « dégpût »/《 dégoûté »+ -tu (nakhaiztu), nekhe « peine »/《 pénible», ohi « habituel »/« habitude »+-tu, oiher « tortueux, sinistre $» / \ll$ lieu retiré »+ $-t u$, okher « tordu »/《 faute, dommage $»+-t u$, on « bon »/ « satisfaction $»+-t u /$-du, onbehar «nécessiteux »/《 nécessité »+ -tu, ongarri « propre à améliorer »/《 fumier» (cf. ongarriztatu), oso « entier »/《 santé »+ -tu, osatu, pagu $«$ contentement $» / \ll$ content $»+-t u$, peitu « manque $» /$ dépourvu $»+-t u$, pisu « poids »/《lourd» (pisü), + -tü, samin « piquant, dur $» /$ « dureté » $+-t u$, sendo $«$ fort $» / \ll$ force $»+-t u$, suhar « ardent, impétueux »/《 pus, gangrène » + -tu, tinka « serré, pressé »/《 tension, frein »+ $+t u$, *trebes « traverse »/《 épars » (cf trebes (k)atu), urru « gratuit »/《 gratuit »+-tu, ülhün « sombre $» / \ll$ nuit »+-tu, ximur « ride $» / \ll$ ridé $»+-t u$, zabar « paresseux, dépravé $» / «$ débris » $+-t u$, zikin « saleté »/《 sale »+ -tu, zohar « (ciel) serein »/« sérénité (du ciel) + -tu.

Ces 197 items constituent une masse assez impressionnante. Dans leur très grande majorité, c'est surtout leur valeur adjectivale qui permet une dérivation verbale - 164 cas-, indiquée généralement par le suffixe - tu/-du/-tü/-dü (ce deux derniers souletins), suffixe qui, rappelons-le, est absent lorsque le verbe est conjugué avec un « auxiliaire second» *di ( edin ») et *za (" ezan »), le lexème « nu » fonctionnant alors comme verbe aussi bien que dans d'autres contextes il fonctionnerait comme substantif ou adjectif-sinon adverbe, dans quelques cas.

Quant à savoir pourquoi le dictionnaire de Lhande ne mentionne pas pour tout le lexique basque (essentiellement «septentrional ») que les lexèmes précités comme susceptibles d'assumer les ces deux (sinon trois, parfois quatre) fonctions, cette limitation est due de toute évidence soit à la sémantique - comment « maison », etxe, pourrait-il acquérir une valeur adjectivale ? Il est vrai que le chinois 
pourrait en remontrer au basque en l'occurrence !-, soit à l'« usage »- p. ex. préférence pour dériver un synonyme...

Même si elle se borne à cela, nous pensons que notre réflexion peut renouveler dans une certaine mesure notre approche du lexique bāsque : ce sera un effort supplémentaire pour nous débarrasser des réflexes romanisants, latinisants, indoeuropéanisants. Nous sommes avec le basque dans un autre univers linguistique, qu'il faudra aborder comme tel. Des problèmes semblables pourront surgir quant aux notions - par exemple- de suffixation et de composition : pourquoi écrire gizonagaz comme gizonarekin, alors que ces formes ont respectivement des pluriels gizonakaz et gizonekin ? Ce n'est pas le même schéma morphologique (le " dévoisement » d'une consonne sourde intérieure opéré pour opposer un pluriel à un singulier est une monstruosité : il s'agit bien de gizona gaz et gizonak gaz, n'en déplaise à nos grammairiens normatifs) ; de même pour les types semea(ren)gatik, ou semea(ren)gan; où il est inadmissible que l'on puisse suffixer une forme déterminée : écrit-on *etxearenondoan ou *oihanarenalde alors qu'ondo-même si le terme n'apparaît que suffixé - et alde sont des substantifs autonomes, juxtaposés à leur expansion? Les types aitarentzat ou aitarekin, même s'ils sont primitivement dérivés du « génitif possessif », ne posent pas aujourd'hui le même problème, puisque ni $t z a t$ ni kin ne sont des mots autonomes - peut-être en allait-il différemment à l'origine...

Ces dernières réflexions ont bien à voir avec le sujet de notre article : l'analyse du lexique basque est à mener non seulement selon les dictionnaires antérieurement constitués ou les descriptions grammaticales (plus ou nooins normatives) proposées par nos prédécesseurs. En basque, qu'est-ce qu'un adjectif ? un substantif? un adverbe ? un suffixe cf. la Morfologia vasca d'Azkue- ? une «postposition »? et même un verbe (en dehors des auxiliaires fléchis) ? Ce n'est qu'après avoir procédé à une nouvelle analyse des composantes du lexique et de l'arsenal des signifiants fonctionnels que l'on pourra enfin décrire le basque pour lui et par lui-même. 